\title{
Initial treatment pattern and prognosis of exocrine pancreatic cancer in Korea from 2006 to 2017
}

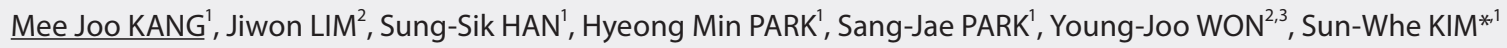 \\ ${ }^{1}$ Center for Liver and Pancreatobiliary Cancer, National Cancer Center, Goyang, Korea \\ ${ }^{2}$ Division of Cancer Registration and Surveillance, National Cancer Center, Goyang, Korea \\ ${ }^{3}$ Department of Cancer Control \& Population Health, Graduate School of Cancer Science and Policy, National Cancer Center, Goyang, Korea
}

Introduction: Hospital-based database had limitations in holistic analysis of cancer treatment and prognosis, omitting those who are out-of-hospital. In addition, elderly patients with pancreatic cancer were under-represented in clinical studies.

Methods: "First course of treatment (FT)" recorded by cancer registries refers to actually administered treatments within four months of diagnosis. Trends in initial treatment pattern and resultant prognosis of exocrine pancreatic cancer (EPC) in Korea from 2006 to 2017 were investigated based on data of the Korea Central Cancer Registry.

Results: Of all 62,209 EPC cases, localized and regional SEER stage (LR), patients over 70 years-old, and adenocarcinoma and its variants (ADC) accounted for 40.6\%, 50.1\%, and 95.9\%, respectively. The most frequent FT was "no active treatment (NAT)" (46.5\%), followed by chemotherapy with/without/or radiotherapy (28.7\%) and surgery (22.0\%). Among 25,198 LR EPC patients, surgical FT increased from $35.9 \%$ to $46.3 \%$ and NAT decreased from $45.0 \%$ to $29.5 \%$. NAT rate was higher in octogenarian (81.2\%) and septuagenarian (44.7\%) compared to those younger than 70 years-old $(19.6 \%, p<0.001)$. Patients with LR ADC had lower rate of surgery $(39.6 \%)$ compared to cystic cancer variants $(86.7 \%)$ or acinar cell carcinoma $(82.8 \%, p<0.001)$. Five-year relative survival rates of LR ADC were higher after surgery (46.1\% [localized], $23.6 \%$ [regional]) compared to NAT (12.9\%, 4.9\%, respectively).

Conclusions: Over $55 \%$ of patients with LR EPC in their 70 s or 80 s do not receive active treatment within four months of diagnosis. Clinicians should pay attention to elderly EPC patients to provide proper medical advice. 